\title{
Influence of metallic and dielectric nanowire arrays on the photoluminescence properties of P3HT thin films
}

\author{
M Handloser ${ }^{1}$, R B Dunbar ${ }^{2}$, A Wisnet ${ }^{1}$, P Altpeter $^{2}$, C Scheu ${ }^{1}$, \\ L Schmidt-Mende ${ }^{3}$ and A Hartschuh ${ }^{1}$ \\ ${ }^{1}$ Department of Chemistry and CeNS, Ludwig-Maximilians-Universität München, 81377 Munich, \\ Germany \\ ${ }^{2}$ Department of Physics and CeNS, Ludwig-Maximilians-Universität München, 80799 Munich, \\ Germany \\ ${ }^{3}$ Department of Physics, University of Konstanz, 78457 Constance, Germany \\ E-mail: achim.hartschuh@cup.uni-muenchen.de
}

\begin{abstract}
The optical properties of organic semiconductor thin films deposited on nanostructured surfaces are investigated using time-resolved two-photon photoluminescence (PL) microscopy. The surfaces consist of parallel aligned metallic or dielectric nanowires forming well-defined arrays on glass substrates. Keeping the nanowire dimensions constant and varying only their spacing from 40 to $400 \mathrm{~nm}$, we study the range of different types of nanowire-semiconductor interactions. For silver nanowires and spacings below $100 \mathrm{~nm}$, the PL intensity and lifetime of P3HT and MDMO-PPV decrease rapidly due to the short-ranged metal-induced quenching that dominates the PL response with respect to a possible plasmonic enhancement of optical transition rates. In the case of P3HT however, we observe an additional longer-ranged reduction of non-radiative losses for both metallic and dielectric nanowires that is not observed for MDMO-PPV. Excitation polarization dependent measurements indicate that this reduction is due to self-assembly of the P3HT polymer chains along the nanowires. In conclusion, nanostructured surfaces, when fabricated across large areas, could be used to control film morphologies and to improve energy transport and collection efficiencies in P3HT-based solar cells.
\end{abstract}

\section{Introduction}

Organic polymers find wide application in electronics and optoelectronics due to their flexibility, low cost and non-toxicity, and appealing optical properties [1]. A large number of polymer-based devices have been developed recently such as solar cells, transistors and organic light emitting diodes (OLEDs). For organic solar cell applications,
P3HT (poly3-hexylthiophene) in combination with PCBM ([6,6]-phenyl $\mathrm{C}_{61}$-butyric acid methylester) has been a standard material for many years, because of its superior charge transport properties and high absorption coefficient (around $10^{5} \mathrm{~cm}^{-1}$ ) in thin films as compared to other organic polymers [2]. Photoabsorption in conjugated polymers such as P3HT leads to the generation of singlet excitons with binding energies of $0.3-1 \mathrm{eV}$ [2]. To increase the efficiency of these 
solar cells further and to make them more competitive with inorganic devices, a major disadvantage of polymer materials has to be overcome: the short exciton diffusion length on the order of $10 \mathrm{~nm}$ that is substantially smaller than the absorption length [3]. Present device designs thus need to balance energy transport and light absorption efficiencies limiting present applications in the case of P3HT to thin films only [4].

Several strategies are currently being investigated that focus either on controlling the film morphology or on enhancing light absorption. Increased polymer chain ordering is expected to improve energy transport properties by ensuring uninterrupted percolation pathways [5, 6]. Zhao et al showed that by combining P3HT with a specially functionalized fullerene electron acceptor and using an optimized annealing treatment, a power conversion efficiency of $6.48 \%$ can be reached, representing the highest value reported for P3HT-based polymer solar cells at that time [7]. Film morphologies can also be influenced by tailoring the surface chemistry of the metal-oxide electrode $[8,9]$ or by fabricating nanostructured heterojunctions using nanoporous metal films [10, 11]. Light absorption, on the other hand, can be enhanced locally by introducing plasmonically active metal nanoparticles or metal nanostructures into the film, permitting a considerable reduction in the physical thickness of the absorbing polymer layers [12-17].

In this work, we investigate the influence of parallel aligned nanowires on the photoluminescence (PL) characteristics of P3HT thin films using time-resolved PL microscopy and spectroscopy. To separate and identify possible plasmonic effects on the optical transition rates, we compare the PL response for identical array configurations comprising either metallic (Ag) or dielectric (glass) nanowires. Two-photon excitation of P3HT at $800 \mathrm{~nm}$ by a pulsed laser source was used to exploit the plasmon resonance of silver at the two-photon transition [18]. The distance dependence of the nanowire-polymer interactions was investigated by varying the nanowire spacing. This approach uses the reduced average nanowire-polymer distance to separate different types of nanowire-polymer interactions and their range. Identical nanostructures using MDMO-PPV (poly(2-methoxy-5-(3'-7'dimethyloctyloxy)-1,4-phenylenevinylene)) were studied for comparison. For the present silver nanowires, the PL response is observed to be dominated by short-ranged metal-induced quenching rather than plasmonic enhancement of optical transition rates. Surprisingly, P3HT shows a substantial reduction of non-radiative losses for both metallic and dielectric nanowires in the case of parallel laser polarization that is not observed for MDMO-PPV. Excitation polarization dependent measurements indicate that this reduction is due to self-assembly of the P3HT polymer chains along the nanowires.

\section{Materials and methods}

Nanowires with a width of $100 \mathrm{~nm}$, a height of $40 \mathrm{~nm}$ and a length of $10 \mu \mathrm{m}$ were fabricated in $10 \mu \mathrm{m} \times$ $10 \mu \mathrm{m}$ arrays on standard microscope glass coverslides by electron-beam lithography. The spacing between the
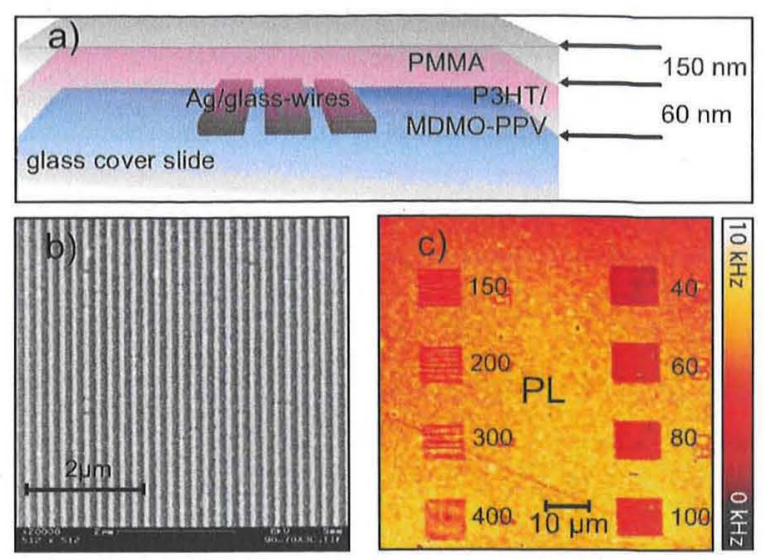

Figure 1. (a) Schematic of the sample layout. (Dimensions not to scale.) (b) Scanning electron microscope image of an Ag nanowire array. (c) PL image of Ag nanowire arrays coated with a P3HT thin film after two-photon laser excitation at $800 \mathrm{~nm}$ detected at higher photon energies. The nanowire spacing decreases from $400 \mathrm{~nm}$ (bottom left) to $40 \mathrm{~nm}$ (top right). Since decreasing nanowire spacing results in a reduced average nanowire-polymer separation, different distance dependent nanowire-polymer interactions can be separated by monitoring PL intensities and lifetimes. The small numbers on the right side of each array indicate the nanowire separation $(x \mathrm{~nm})$.

nanowires was systematically varied from array to array between 40 and $400 \mathrm{~nm}$. On top of the structures a thin polymer film of either P3HT or MDMO-PPV was spin-coated from a chlorobenzene solution with a nominal thickness of $60 \mathrm{~nm}$ measured by AFM. For all samples, the same spin coating procedure with the same volume of polymer solution was used. Previous studies in the literature indicate that spin coating of P3HT and other polymers from solution onto different nanostructured surfaces leads to the filling of pores and gaps down to dimensions of about $5 \mathrm{~nm}[19,20]$. We thus assume uniform sample height and complete filling of the volume in between the nanowires by the polymer, as shown in the schematic in figure 1(a). For this sample geometry, a reduction of the nanowire spacing is expected to be connected to a decrease of the polymer volume within the focus of the confocal microscope used for the optical measurements. This decrease in volume can be calculated using the geometrical parameters of the sample. At the smallest spacing of $40 \mathrm{~nm}$ of the $100 \mathrm{~nm}$ wide nanowires, the pitch of the structure is $140 \mathrm{~nm}$. Within the focal diameter of $400 \mathrm{~nm}$ (see below) we thus observe about four nanowires. The ratio between the volume filled by the nanowires and the total sample volume within the focus with a sample thickness of $60 \mathrm{~nm}$ is thus $(4 \times 40 \mathrm{~nm} \times 100 \mathrm{~nm}) /(400 \mathrm{~nm} \times 60 \mathrm{~nm})=2 / 3$. As a result, a volume related linear decrease of the PL intensity by up to a factor of $2 / 3$ is expected upon decreasing nanowire spacings.

Finally, the samples were top-coated with a PMMA film of $150 \mathrm{~nm}$ thickness to reduce oxidation and to increase the photochemical stability of the organic polymers. Figure 1(a) presents a schematic of the sample configuration together with a representative scanning electron microscope (SEM) image in figure 1(b), showing a silver nanowire array with a nanowire spacing of $100 \mathrm{~nm}$. Further details on the sample 
preparation are provided in the supporting material (available at stacks.iop.org/Nano/23/305402/mmedia) together with additional topography and SEM data of the nanowires (figures $\mathrm{S} 1$ and $\mathrm{S} 2$ available at stacks.iop.org/Nano/23/ 305402/mmedia).

Time-correlated single photon counting (TCSPC) was used in combination with confocal microscopy for timeresolved PL measurements. In the studied polymers, PL results from radiative exciton recombination and its intensity $I_{\mathrm{PL}}$ is proportional to the product of the absorbed photon flux $I_{\mathrm{L}}$ and the PL quantum yield $Q_{\mathrm{PL}}$ of the polymer $I_{\mathrm{PL}} \propto I_{\mathrm{L}} Q_{\mathrm{PL}}$. The quantum yield is defined as the ratio between the radiative decay rate $k_{\text {rad }}$ and the sum of radiative and non-radiative decay rates $k_{\text {non-rad }}$ corresponding to $Q_{\mathrm{PL}}=k_{\mathrm{rad}} /\left(k_{\mathrm{rad}}+\right.$ $\left.k_{\text {non-rad }}\right)=k_{\text {rad }} \tau$. Here, we expressed the sum of all decay rates $\tau=1 /\left(k_{\text {rad }}+k_{\text {non-rad }}\right)$ by the excited state lifetime $\tau$. The combination of PL intensity and lifetime measurements thus provides access to changes in the excited state relaxation and helps to distinguish radiative and non-radiative rate modifications.

Laser excitation was provided by a Ti:sapphire oscillator operating at a photon energy of $1.55 \mathrm{eV}(800 \mathrm{~nm})$, a repetition rate of $76 \mathrm{MHz}$ and a pulse duration of $150 \mathrm{fs}$ with linear polarization. The PL signal was detected at higher photon energies between 550 and $750 \mathrm{~nm}$ corresponding to two-photon excitation [21]. Since two-photon transition probabilities scale with the square of the local intensity, they are well suited to study plasmonically enhanced local light fields. Here, we aim at exploiting the plasmon resonance of silver at the two-photon transition. Laser illumination of the metal nanowire ends is expected to excite both localized surface plasmons confined to the tips as well as propagating surface plasmon modes for light polarized along the nanowire axis $[15,18,22]$. In contrast, perpendicular polarization and excitation along the nanowires will result in a weaker response due to a reduced lightning rod effect and the momentum mismatch between propagating light waves and surface plasmons, respectively.

Laser polarization along and perpendicular to the nanowires is obtained by rotating the sample with respect to the linearly polarized source. This procedure avoids signal variations due to polarization dependent optical components in the excitation and detection path. PL detection is non-polarized in all measurements. The inverted confocal microscope used in epi detection combined with a high numerical aperture objective $(\mathrm{NA}=1.3)$ provides a diffraction limited excitation spot of about $400 \mathrm{~nm}$. Due to the high NA and the associated large angular range of excitation and collection, we do not expect significant angular dependent grating effects on the detected PL intensities and lifetimes. A fast avalanche photodiode (APD) was used to detect PL transients and intensities. The instrument response function (IRF) of the system was independently measured by detecting the scattered pump laser light from the sample and had a full width at half maximum (FWHM) of $27 \mathrm{ps.} \mathrm{Due} \mathrm{to} \mathrm{the}$ high signal to noise ratio achieved in our experiments and the reproducibility of the IRF the time resolution of the setup is about 3 ps, close to $10 \%$ of the FWHM of the IRF [23]. All
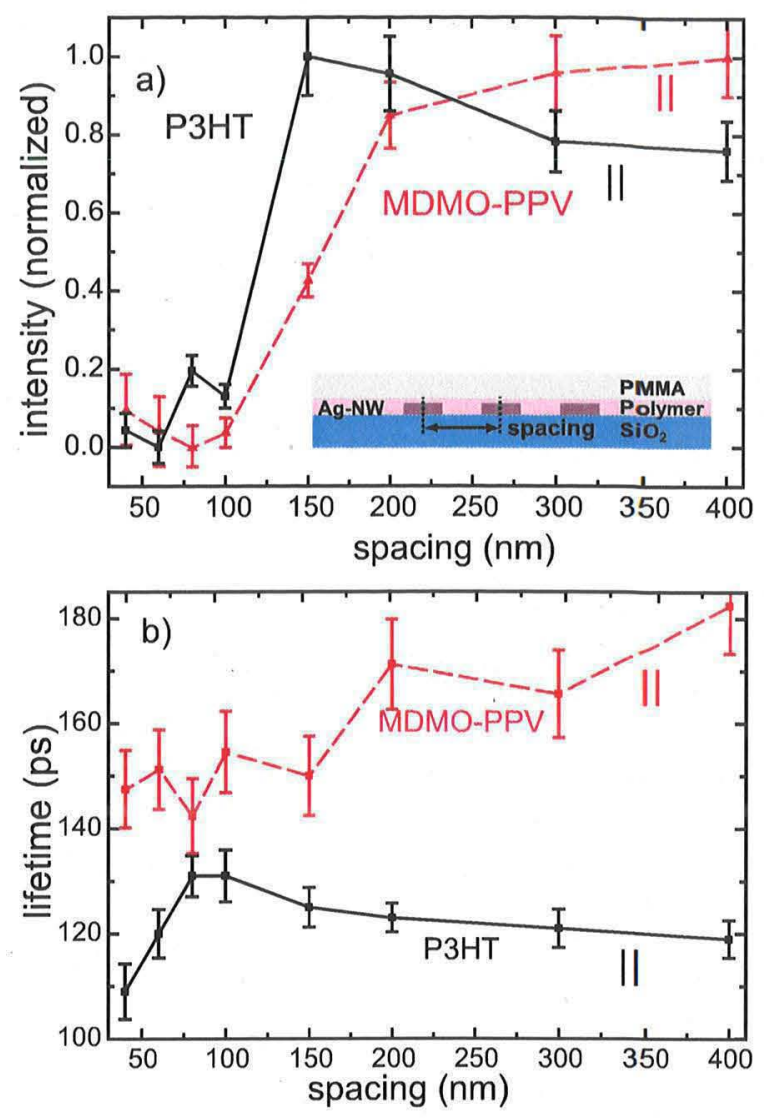

Figure 2. PL intensities (a) and intensity-weighted average lifetimes (b) recorded for P3HT (black solid curves) and MDMO-PPV (red dashed curves) for varying silver nanowire spacing. The PL intensity of P3HT is seen to increase slowly and to peak at about $150 \mathrm{~nm}$ while MDMO-PPV shows a continuous decrease for decreasing nanowire spacing. A corresponding trend is observed in the PL lifetimes while the peak of P3HT shifted to smaller nanowire spacing. The intensities in (a) are normalized with respect to the intensity measured next to the nanowire array of the respective polymer film. The polarization of the exciting laser light was parallel to the nanowires in (a) and (b).

recorded photoluminescence transients have been modeled using bi- or tri-exponential fit functions. From the fit results, intensity averaged PL lifetimes $\tau_{\mathrm{IA}}$ are calculated as $\tau_{\mathrm{IA}}=$ $\sum_{n}\left(A_{n} \tau_{n}^{2}\right) /\left(A_{n} \tau_{n}\right)$. Here $A_{n}$ denotes the amplitude of the $n$th exponential decay component and $\tau_{n}$ the associated lifetime.

A representative PL image of silver nanowire arrays with different nanowire spacings covered by a P3HT film with a nominal thickness of $60 \mathrm{~nm}$ is shown in figure 1(c). Here the nanowire separation is reduced from bottom left to top right from 400 to $40 \mathrm{~nm}$. To minimize fluctuations due to sample inhomogeneities, PL transients and intensity measurements were performed at several points, typically 30-50, within an array of fixed nanowire distances.

\section{Results and discussion}

The PL intensities and lifetimes of P3HT and MDMO-PPV for varying silver nanowire spacing are shown in figures 2(a) 
and (b), respectively. The intensity data in figure 2(a) are normalized to the values of the studied planar polymer films measured at a short distance away from the arrays where the substrate surface is flat (an example PL image is shown in figure 1(c)). For both materials, the PL intensity decreases rapidly for nanowire spacings below $150 \mathrm{~nm}$ reaching nearly zero at about $60 \mathrm{~nm}$ (figure 2(a)). This decrease is substantially larger than the factor $2 / 3$ that would be expected from a pure volume effect (see section 2 above). Remarkably, the PL intensity of P3HT peaks at about $150 \mathrm{~nm}$ while for MDMO-PPV it decreases monotonously with decreasing spacing. A corresponding peak at slightly smaller spacings is also seen in the lifetime data for P3HT (figure 2(b)). The lifetimes observed for MDMO-PPV, on the other hand, are seen to drop rather monotonously with decreasing spacing carrying large uncertainties due to sample inhomogeneities, faster photobleaching and reduced signal levels in particular for spacings below $200 \mathrm{~nm}$.

From the decreasing PL intensities and lifetimes for very small spacing we conclude that for both P3HT and MDMOPPV a short-ranged interaction with the metallic nanowires becomes dominant representing an efficient non-radiative relaxation channel. The slow increase with decreasing spacing and peak in the P3HT intensity and lifetime data, on the other hand, reveals the presence of an additional longer-ranged interaction mechanism that leads to the reduction of a non-radiative relaxation channel. The origins of these two different types of nanowire-polymer interactions are the subject of the following studies.

The short-ranged decrease of the PL intensity occurring for both polymers can be attributed to energy transfer from the emissive exciton state to the Ag nanowires that is well known from the literature for metal substrates (e.g. [24]). To determine the range of metal-induced quenching in our samples, we prepared MDMO-PPV and P3HT films of various thicknesses on a thin silver film $(\approx 20 \mathrm{~nm})$. The results (figure 3 ) show that quenching plays a dominant role up to a film thickness of about $40-50 \mathrm{~nm}$ after which the lifetime observed for pristine polymer films is reached (red solid line in figure 3). The lifetimes shown in figure 3 are intensity-weighted average values that are determined by the interplay between energy transfer within the film and to the metal substrate that is followed by non-radiative decay [24, 25]. The measured PL lifetime of the unstructured P3HT thin film measured on glass for reference is comparable to the values reported in the literature and are somewhat shorter than those observed in solution [26-28]. Similar PL lifetimes were observed for the same sample positions upon one- and two-photon excitation at $530 \mathrm{~nm}$ and $800 \mathrm{~nm}$, respectively (figure S3 in the supporting material available at stacks.iop. org/Nano/23/305402/mmedia).

The longer-ranged increase of the PL intensity and lifetime observed for P3HT in figure 2 is unexpected. To determine its origin, we first studied the polarization dependence of the PL characteristics. The spacing dependent PL lifetimes and intensities of P3HT for two orthogonal laser polarizations are shown in figure 4 . To verify the reproducibility of the features observed in figure 2 , this


Figure 3. Metal-induced PL quenching studied by varying the thickness of the polymer film deposited on a thin silver layer $(20 \mathrm{~nm})$ for (a) P3HT and (b) MDMO-PPV. Both polymers show efficient metal-induced quenching up to a distance of about $40-50 \mathrm{~nm}$.

data has been recorded on a different silver nanowire array sample with the same structural parameters. In figure 4, the polarization of the excitation laser is changed from parallel (black solid curve) to perpendicular (green dashed curve) with respect to the nanowire orientation. For perpendicular polarization, no change in lifetime and intensity can be detected within the error bars of both quantities besides the short-ranged metal-induced quenching for separations below $150 \mathrm{~nm}$ discussed above. Parallel polarization, on the other hand, results in the same spacing dependence as observed in figure 2 for the same polarization with somewhat different absolute numbers due to sample-to-sample variations.

Since the lifetime increases in the presence of the metal nanowire, we can rule out radiative rate enhancement due to coupling to plasmons as the reason for the increased PL intensity for parallel polarization. We also do not observe increased PL intensities at the ends of the metal nanowires for which the strongest local plasmon fields would be expected as discussed above in section 2 (figure 1(c)). The transition dipole moment of the lowest optical transition in P3HT is parallel to the polymer chain, connecting the thiophene rings. Polarized excitation is thus able to select differently oriented polymer chains within the film. The longer lifetimes observed 

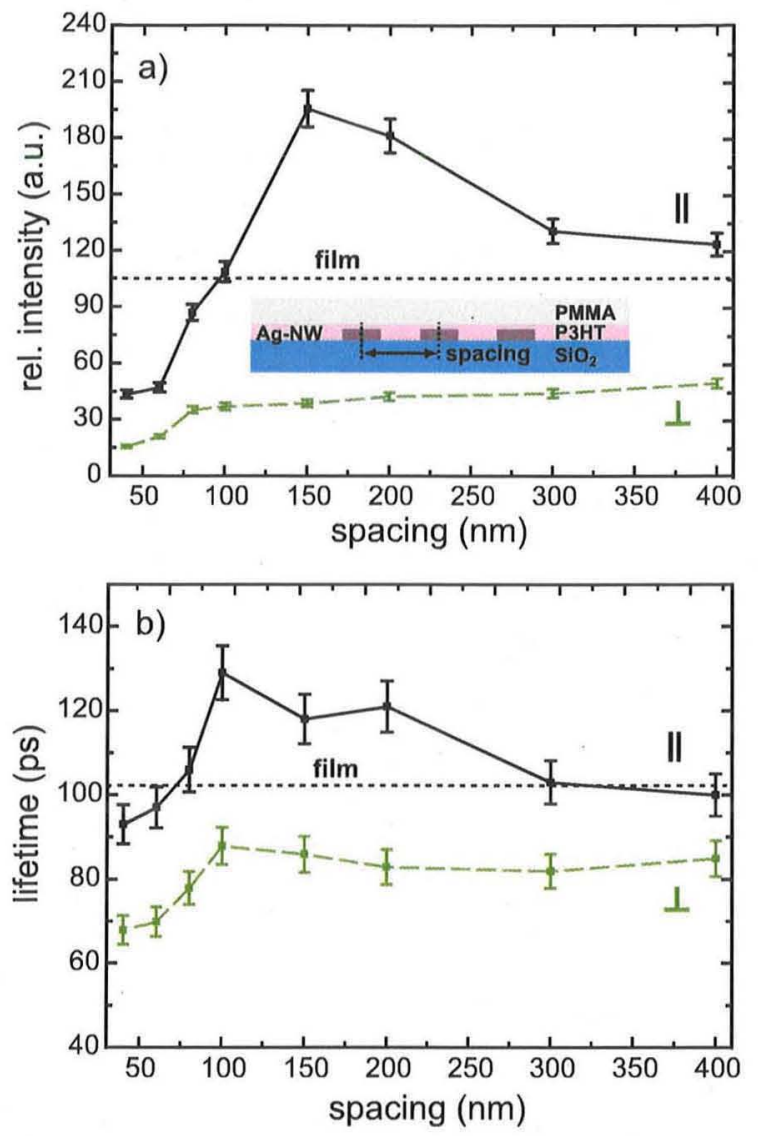

Figure 4. Excitation polarization dependence of PL intensity (a) and lifetime (b) for P3HT covered silver nanowire arrays with different spacings. Black solid and green dashed curves have been recorded at the same sample position for parallel and perpendicular excitation polarization with respect to the nanowires, respectively. The PL intensity data obtained for parallel polarization show the competition between short-ranged metal-induced quenching and a longer-ranged effect that leads to increased PL due to reduced non-radiative losses, as can be seen from simultaneously increased decay times. This increase is not observed for perpendicular polarization and is attributed to nanowire-induced ordering of the polymer chains along the nanowires (see text). Note that since at least one nanowire remains in the focal area even for large separations of $400 \mathrm{~nm}$, the PL characteristics obtained for the two polarization directions need not to be the same.

for parallel excitation indicate that the polymer fraction oriented along the nanowires at a close distance feature reduced non-radiative losses. Since no substantial variations are observed for excitation polarization perpendicular to the nanowires, losses are reduced only for polymer chain orientation along the nanowire. From the literature it is known that for P3HT films ordering of the polymer chains reduces non-radiative losses leading to longer lifetimes [6, 29]. We thus attribute the longer-ranged increase of PL intensity and lifetime to nanowire-induced ordering of the P3HT chains along the nanowires.

To test this hypothesis, we first recorded the corresponding PL intensity and lifetime data of MDMO-PPV on Ag nanowires (data not shown). For this material, we do not

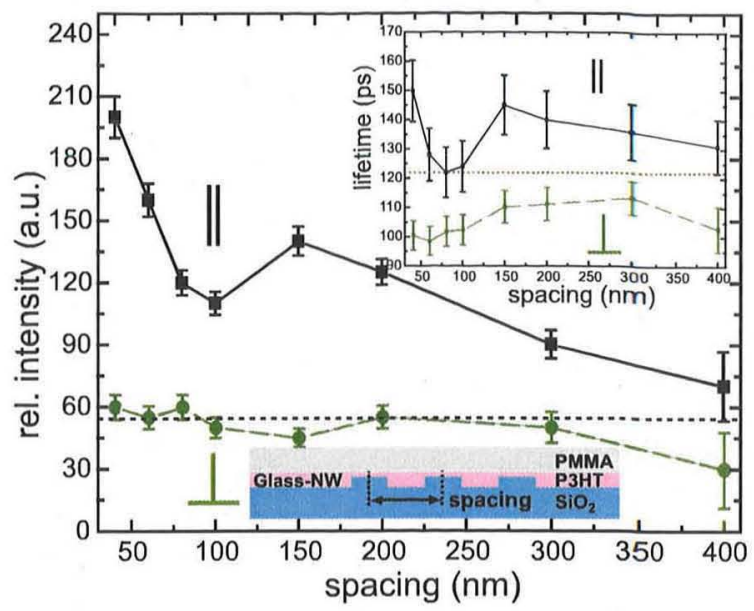

Figure 5. Polarization dependent PL intensity measurement of P3HT on dielectric (glass) nanowire arrays. The black solid curve represents parallel polarization, while the green dashed curve corresponds to perpendicular polarization with respect to the nanowires. PL lifetimes for both polarizations are shown in the inset. The dotted line shows the PL intensity and lifetime value for the unstructured P3HT film measured close to the structured area. Upon decreasing nanowire spacing both PL intensity and lifetime are seen to increase for parallel excitation polarization that is attributed to ordering of the polymer chains induced by the nanostructured surface.

observe a substantial polarization contrast. This indicates that either ordering along the nanowires or the correlation between ordering and non-radiative losses is less pronounced for MDMO-PPV than would be expected from the literature $[30,31]$.

In the next step and to exclude plasmonic effects induced by the metallic nanowires, we fabricated identical nanowire arrays but replacing the silver by a dielectric, $\mathrm{SiO}_{2}$. The polarization dependent PL lifetimes and intensities of P3HT on dielectric nanowire arrays with varying spacings are shown in figure 5. For parallel polarization (black solid curve in figure 5(a)), a substantial increase of the PL intensity is observed upon decreasing nanowire spacing. The range and magnitude of this increase is comparable to the one observed for the metallic nanowire array in figure 2(a). The simultaneous increase in PL lifetime indicates that the intensity variations are due to modified excited state relaxation dynamics within the film and are not caused by an increased material volume. In addition, a decreased nanowire spacing would rather be expected to reduce the amount of P3HT within the focal volume leading to weaker PL assuming a uniform sample height. The origin of the dip seen in both PL intensity and lifetime data around $100 \mathrm{~nm}$ nanowire spacing for parallel polarization is not clear and could reflect sample heterogeneities.

Importantly, no substantial intensity variations for perpendicular polarization (green dashed curve in figure 5) are observed as in the metal case for larger nanowire spacings. To conclude, our data show the same range and polarization dependence of the longer-ranged effect for dielectric and metallic nanowires. Its origin can hence not be plasmonic. 
Instead, these findings support the idea of nanowire-induced ordering of $\mathrm{P} 3 \mathrm{HT}$ as the underlying mechanism for the PL and lifetime increase for smaller nanowire separations developed above. We note that substrate-induced ordering of P3HT has been observed before for samples fabricated by nanoimprint lithography and has been studied theoretically $[32,33]$.

For the present metallic nanowire structures, no substantial plasmonic absorption enhancement can be deduced from our PL intensity and lifetime data. In particular, no increased PL is observed at any point in the PL images of the arrays including the nanowire ends for which the strongest local fields would be expected as discussed in section 2 (figure 1(c)). Due to the complexity of the studied system, which involves intra-film exciton energy transfer, intrinsic non-radiative losses and distance dependent energy transfer to the metallic nanowires, a complete quantitative description or modeling of both PL intensity and lifetime is not feasible. In general, metal-induced quenching appears to be the dominant process as compared to absorption enhancement.

\section{Conclusion}

In summary, we studied the influence of nanowire arrays on the optical properties of P3HT and MDMO-PPV thin films using time-resolved photoluminescence microscopy. Possible plasmonic light absorption enhancement provided by the metallic nanowires is dominated by metal-induced quenching. On the other hand, the results obtained for P3HT films indicate a pronounced ordering effect induced by both metallic and dielectric nanowires reaching up to about $100 \mathrm{~nm}$ that leads to a substantial reduction in non-radiative losses that should result in improved energy transfer efficiencies within the polymer film. Our findings underline the importance of the film morphology and its control for the optimization of polymer solar cells.

\section{Acknowledgment}

We acknowledge financial support by the Deutsche Forschungsgemeinschaft provided through the Nanosystems Initiative Munich (NIM).

\section{References}

[1] Brabec C, Dyakonov V, Parisi J and Sariciftci N S (ed) 2007 Organic Photovoltaics 2 edn (Berlin: Springer)

[2] Zhokhavets U, Erb T, Gobsch G, Al-Ibrahim M and Ambacher O 2006 Chem. Phys. Lett. 418 347-50

[3] Kroeze J E, Savenije T J, Vermeulen M J W and Warman J W 2003 Phys. Chem. B 107 7696-705
[4] Duche D, Drouard E, Simon J, Escoubas L, Torchio P, Le Rouzo J and Vedraine S 2011 Sol. Energy Mater: 95 18-25

[5] Oosterhout S D, Wienk M M, Bavel S S, Thiedmann R, Koster L J A, Gilot J, Loos J, Schmidt V and Janssen R A J 2009 Nature Mater. 8 818-24

[6] Marsh A R, Hodgkiss J M, Albert-Seifried S and Friend R H 2010 Nano Lett. 10 923-30

[7] Zhao G, He Y and Li Y 2010 Adv. Mater. 22 4355-8

[8] Hsu J W P and Lloyd M T 2010 MRS Bull. 35 422-8

[9] Chen D, Nakahara A, Dongguang W, Nordlund D and Russel T P 2011 Nano Lett. 11 561-7

[10] McGehee M D 2009 MRS Bull. 34 95-100

[11] Calhoun M F 2008 Nature Mater. 7 84-9

[12] Atwater H A and Polman A 2010 Nature Mater: 9 205-13

[13] Dunbar R B, Hesse H C, Lembke D S and Schmidt-Mende L 2012 Phys. Rev. B 85035301

[14] Mahmoud M A, Poncheri A J, Phillips R L and El-Sayed M A $2010 \mathrm{~J}$. Am. Chem. Soc. 132 2633-41

[15] Schuller J A, Barnard E S, Cai W, Chul Jun Y, White J S and Brongersma M L 2010 Nature Mater: 9 193-204

[16] Wang D H, Park K H, Seo J H, Seifter J, Jeon J H, Kim J, Park J H, Park O O and Heeger A 2011 Adv. Eng. Mater. $1766-70$

[17] Ferry V E, Sweatlock L A, Pacifici D and Atwater H A 2008 Nano Lett. 8 4391-7

[18] Imura K, Kim Y C, Kim S, Jeong D H and Okamoto H 2009 Phys. Chem. Chem. Phys. 11 5876-81

[19] Coakley K M and McGehee M D 2004 Chem. Mater. $164533-42$

[20] Coakley K M and McGehee M D 2003 Appl. Phys. Lett. 83 3380-2

[21] Trotzky S, Hoyer T, Tuszynski W, Lienau C and Parisi J 2009 J. Phys. D. Appl. Phys. 42055105

[22] Dittlbacher H, Hohenau A, Wagner D, Kreibig U, Rogers M, Hofer F, Aussenegg F R and Krenn J 2005 Phys. Rev. Lett. 95257403

[23] O'Connor D V and Phillips P 1984 Time Correlated Single Photon Counting (London: Academic)

[24] Chance R R, Prock A and Silbey R 1974 J. Chem. Phys. $602184-5$

[25] Gomez U, Port H and Wolf H 1997 J. Lumin. 72-74 496-8

[26] Cook S, Akihiro F and Katoh R 2008 Energy Environ. Sci. 1 294-9

[27] Magnani L, Rumbles G, Samuel I, Murray K, Moratti S, Holmes A and Friend R 1997 Synth. Met. 84 899-900

[28] Kraabel B, Moses D and Heeger A 1995 J. Chem. Phys. 1035102

[29] Labastide J A, Baghgar M, Dujovne I, Venkatraman H B, Ramsdell D C, Venkataraman D and Barnes M D 2011 J. Phys. Chem. Lett. 2 2089-93

[30] van Bavel S, Sourty E, de With G, Frolic K and Loos J 2009 Macromolecules 42 7396-403

[31] Olson D, Piris J, Collins R, Shaheen S and Ginley D 2006 Thin Solid Films 496 26-9

[32] Aryal M, Trivedi K and Hu W 2009 ACS Nano 3 3085-90

[33] Melis C, Colombo L and Mattoni A 2011 J. Phys.Chem. C $115575-81$ 